\title{
Transient scalar hair for nearly extreme black holes
}

\author{
Lior M. Burko $\odot,{ }^{1}$ Gaurav Khanna, ${ }^{2}$ and Subir Sabharwal ${ }^{3}$ \\ ${ }^{1}$ Theiss Research, La Jolla, California 92037, USA \\ ${ }^{2}$ Department of Physics, University of Massachusetts, Dartmouth, Massachusetts 02747, USA \\ ${ }^{3}$ Eastmore Group, 40 Wall Street, New York, New York 10005, USA
}

(Received 7 June 2019; published 15 November 2019)

\begin{abstract}
It has been shown recently that extreme Reissner-Nordström black holes perturbed by a minimally coupled, free, massless scalar field have permanent scalar hair. The hair, a conserved charge calculated at the black hole's event horizon, can be measured by a certain expression at future null infinity: The latter approaches the hair inversely in time. We generalize this newly discovered hair also for extreme Kerr black holes. We study the behavior of nearly extreme black hole hair and its measurement at future null infinity as a transient phenomenon. For nearly extreme black holes the measurement at future null infinity of the length of the newly grown hair decreases quadratically in time at intermediate times until its length becomes short and the rate at which the length shortens further slows down. Eventually, the nearly extreme black hole becomes bald again like nonextreme black holes.
\end{abstract}

DOI: 10.1103/PhysRevResearch.1.033106

\section{INTRODUCTION}

Scalar fields, which are ubiquitous in theoretical physics (e.g., the Higgs field) and in astrophysics (e.g., the inflaton, certain dark matter, and dark energy models), have been proposed as candidates for black hole (BH) hair [1], in possible violation of the no-hair conjecture. The latter states that all BH solutions of the Einstein-Maxwell equations of general relativity can be completely characterized by three and only three externally observable classical parameters, specifically the BH's mass $M$, charge $q$, and spin angular momentum $a$. Bekenstein provided a proof for the nonexistence of scalar hair given a set of assumptions [2-4]. A number of scalar field hair models have been found, where one or more of the assumptions underlying Bekenstein's theorem are violated. Those include scalar fields with nonstrictly positive potentials, scalar fields which are noncanonical or nonminimally coupled to gravity, bound states of "bald" BHs and solitons [1], or spacetimes with more than four dimensions [5]. Also, nonscalar field hair models have been suggested, including non-Abelian Yang-Mills [6] or Proca fields [7]. In all these examples it is the field itself that constitutes the BH's hair. In addition, when quantum mechanical effects are included, $\mathrm{BHs}$ can carry quantum numbers [8] and have soft hair [9].

More recently, a different kind of scalar hair for extreme Reissner-Nordström (ERN) BHs was found by Angelopoulos, Aretakis, and Gajic (AAG) [10], where a certain quantity $s[\psi]$ evaluated at future null infinity $\left(\mathscr{I}^{+}\right)$equals a nonvanishing quantity $H[\psi]$ - which may be called the Aretakis charge,

Published by the American Physical Society under the terms of the Creative Commons Attribution 4.0 International license. Further distribution of this work must maintain attribution to the author(s) and the published article's title, journal citation, and DOI. the AAG hair, or the horizon integral - that is calculated on the BH's event horizon (EH), but vanishes if the $\mathrm{BH}$ is nonextreme. Since $H[\psi]$ is a conserved charge for ERN BHs [11], it would naturally be related to a candidate for $\mathrm{BH}$ hair. Indeed, in [10] it was shown that $s[\psi]$ equals $H[\psi]$. The AAG hair may be construed as a different class of $\mathrm{BH}$ hair than the types of hair discussed above, as it is made of a minimally coupled, free, massless scalar field. However, it is not the scalar field itself which constitutes the measurement at $\mathscr{I}^{+}$of the AAG hair, but a functional of the scalar field $\psi$ which is calculated by adding two terms evaluated at $\mathscr{I}^{+}$, an asymptotic term $s_{\mathrm{I}}[\psi]$ and a global term $s_{\mathrm{II}}[\psi]$,

$$
s[\psi]:=\frac{1}{4 M} \lim _{u \rightarrow \infty} u^{2} \times(r \psi)+\frac{1}{8 \pi} \int_{\mathscr{I}^{+} \cap\{u \geqslant 0\}}(r \psi) d \Omega d u,
$$

where $\psi$ is evaluated on $\mathscr{I}^{+}\left(\left.\psi\right|_{\mathscr{I}^{+}}\right)$and $u$ is retarded time. Angelopoulos, Aretakis, and Gajic showed that $s[\psi]=H[\psi]$ for ERN BHs, but $s[\psi]=0$ for nonextreme RN BHs, where

$$
H[\psi]:=-\frac{M^{2}}{4 \pi} \int_{\mathrm{EH}} \partial_{r}(r \psi) d \Omega,
$$

which is calculated on the BH's EH (AAG hair). We evaluate below $s[\psi](u)$ by evaluating $s_{\mathrm{I}}[\psi](u)$ [without taking the limit in Eq. (1)] and by truncating the integration in $s_{\mathrm{II}}[\psi]$ at $u$. We evaluate below $H[\psi](v)$ by integrating separately for each value of advanced time $v$.

In what follows we first verify numerically the occurrence of AAG hair for ERN BHs. We then generalize the AAG hair also for extreme Kerr (EK) BHs. We next consider nearly extreme BHs (NERN or NEK BHs) and show the AAG hair as a transient behavior, including observational features from far away. 

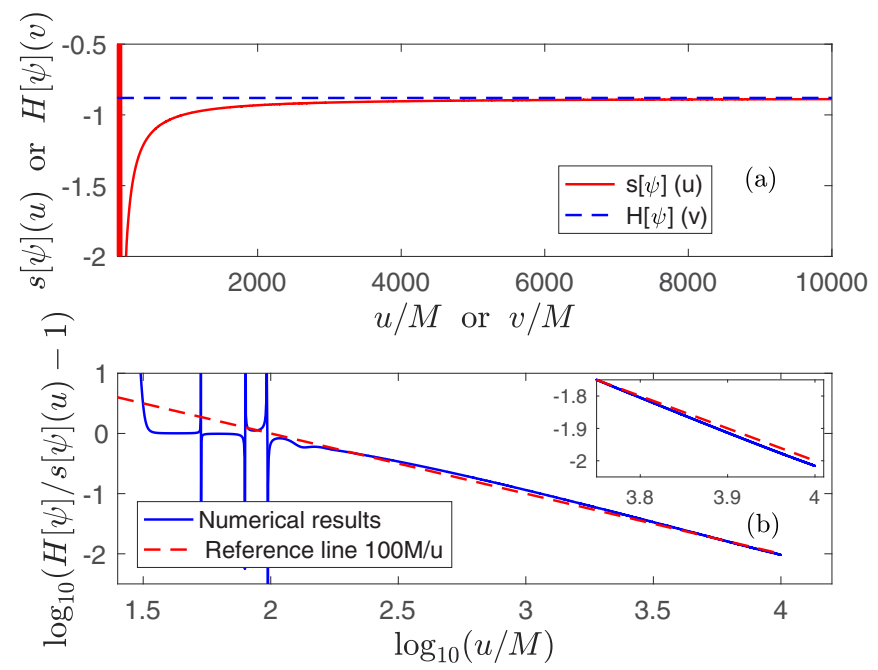

FIG. 1. (a) Measurement at $\mathscr{I}^{+}$of the AAG hair $s[\psi](u)$ (solid curve) and the AAG hair $H[\psi](v)$ (dashed curve), in units of $M^{2}$, as functions of retarded $(u)$ and advanced $(v)$ times, respectively, for ERN. (b) Relative difference between the measurements in (a) when the EH integral is evaluated for $v \gg M$ (solid curve). The dashed curve is the reference curve $100 M / u$.

\section{NUMERICAL METHOD}

Our numerical simulations begin with writing the $(2+$ 1)-dimensional scalar wave equation in Reissner-Nordström (RN) or Kerr spacetime backgrounds (Teukolsky equation) for azimuthal $(m=0)$ modes in compactified hyperboloidal coordinates, which allow us to access $\mathscr{I}^{+}$at a finite radial coordinate [12]. The resulting second-order hyperbolic partial differential equation is then rewritten as a coupled system of two first-order hyperbolic equations. We then solve this system by implementing a second-order RichtmyerLax-Wendroff iterative evolution scheme $[13,14]$. The initial data are a "truncated" Gaussian (to ensure compact support) with nonzero initial field values on the EH. Specifically, in hyperboloidal coordinates $(\rho, \tau)$ (see [13] for definitions), the initially spherical $(\ell=0)$ Gaussian pulse is centered at $\rho=1.0 M$ with a width of $0.22 M$ so that we have horizon penetrating initial data that lead to $H[\psi] \neq 0$ on the initial data surface [10]. (For example, the horizon is at $\rho=0.95 M$ for ERN and EK BHs in these coordinates.) The Gaussian is truncated beyond $\rho=8.0 \mathrm{M}$ and the outer boundary is located at $S=\rho\left(\mathscr{I}^{+}\right)=19.0 \mathrm{M}$.

In practice, we approximate $H[\psi](v)$ with $H[\psi](\tau)$. At finite times the difference between $\tau$ and $v$ (see Fig. 1 in [14]) is manifested in an apparent variation in $H[\psi](v)$ which is a numerical artifact resulting from this approximation. For that reason, the physically relevant value which we use is $H[\psi](v \gg M)$.

\section{EXTREME RN OR KERR BLACK HOLES: NUMERICAL TESTS}

First, we show in Fig. $1 s[\psi](u)$ and $H[\psi](v)$ as functions of $u$ and $v$, respectively, for an ERN BH. Both fields vary as functions of time, although the (unphysical) changes in $H[\psi](v)$ are not visible on the scale of this
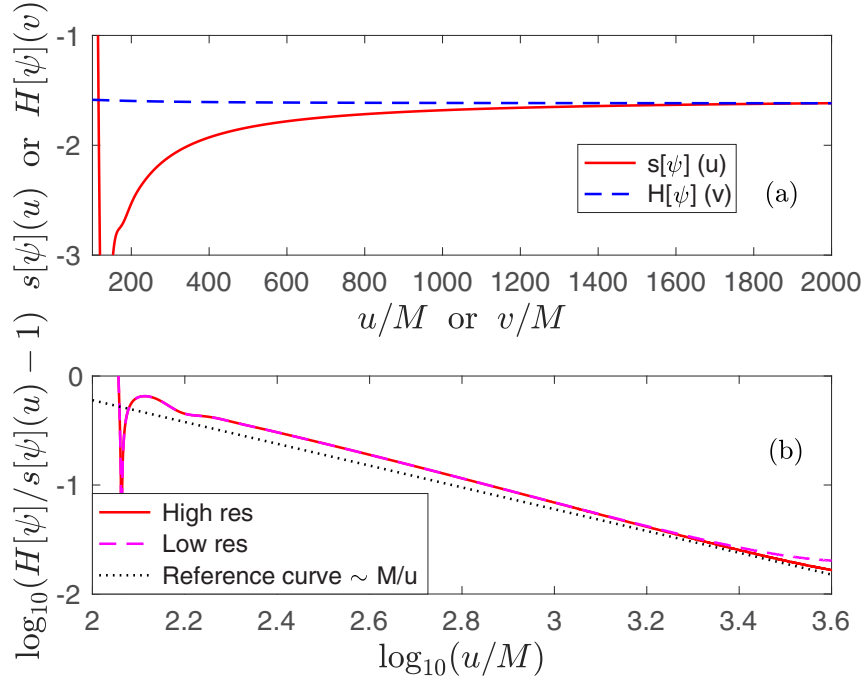

FIG. 2. Same as Fig. 1 for an EK BH. Note that (b) shows the results for both low and high grid resolutions.

figure. Figure 1 also shows the relative difference $H[\psi](v \gg$ $M) / s[\psi](u)-1$ as a function of $u$, where $H[\psi](v \gg M)$ approximates $H[\psi](v \rightarrow \infty)$. We find that $s[\psi](u)$ approaches $H[\psi](v \rightarrow \infty)$ for late $u$ as $1 / u$ (i.e., $s[\psi](u) \sim H[\psi](v \rightarrow$ $\left.\infty)+\mathscr{H}^{\mathrm{RN}}[\psi] / u\right)$. We find for our choice of initial data $\mathscr{H}^{\mathrm{RN}}[\psi] M^{-3} \sim 100 \pm 1$.

We then apply $s[\psi]$ and $H[\psi]$ also for EK BHs and present our results in Fig. 2. Accurate numerical calculation of $H[\psi]$ is more challenging for EK BHs than for ERN BHs and requires us to increase the numerical grid density substantially. Figure 2 shows evidence for AAG hair for EK BHs. We find also for EK BHs that $s[\psi](u)$ approaches $H[\psi](v \rightarrow \infty)$ for late $u$ as $1 / u$ (i.e., $\left.s[\psi](u) \sim H[\psi](v \rightarrow \infty)+\mathscr{H}^{\mathrm{K}}[\psi] / u\right)$. Here $\mathscr{H}^{\mathrm{K}}[\psi] M^{-3} \sim 70 \pm 1$.

\section{NEARLY EXTREME AND NONEXTREME RN OR KERR BLACK HOLES: NUMERICAL RESULTS}

Next we consider NERN and NEK BHs. The AAG hair $H[\psi](v)$ is shown in Fig. 3 for a number of $q / M$ and $a / M$ values for RN and Kerr BHs, respectively. For the extreme cases Fig. 3 shows the respective Aretakis charges [11]. For nonextreme $\mathrm{BHs} H[\psi](v)$ attains vanishing values rapidly. For nearly extreme BHs the $H[\psi](v)$ start at early times with values close to their extreme counterparts, and at late times they approach the nonextreme vanishing values. The closer the $\mathrm{BH}$ to extremality, the longer $H[\psi](v)$ takes to get close to zero.

We expect that for nearly extreme BHs at early times $s[\psi]$ would appear to be similar to that of ERN or EK BHs, but that at late times it would behave similarly to nonextremal BHs. That is, we expect transient growth of the measurement at $\mathscr{I}^{+}$of scalar hair for NERN and NEK BHs, after which they would become bald again. Figure 4 shows $s[\psi](u)$ for a number of $a / M$ values for Kerr BHs and for a number of $q / M$ values for RN BHs. The measurement $s[\psi](u)$ approaches a nonzero constant for extreme BHs as $u \rightarrow \infty$, whereas $s[\psi](u) \rightarrow 0$ for nonextreme BHs. The values of $s[\psi](u)$ for nearly extreme $\mathrm{BHs}$ are close at early times to those of 

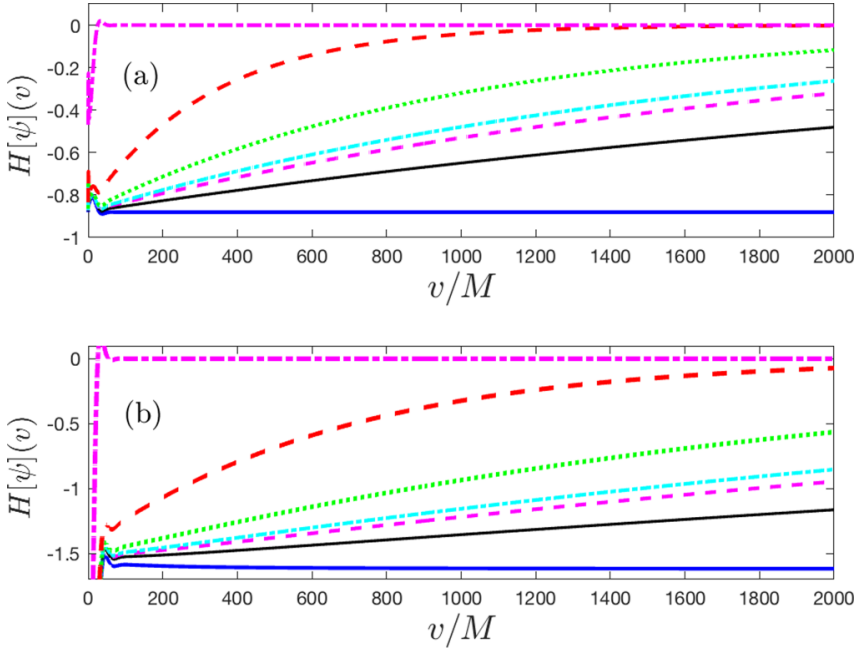

FIG. 3. Horizon integrals $H[\psi](v)$ (in units of $M^{2}$ ) as functions of advanced time $v$ for (a) a number of $1-q / M$ values for RN BHs and (b) a number of $1-a / M$ values for Kerr BHs. In (a), from bottom to top, the values are $1-q / M=0,4.5 \times 10^{-8}, 1.25 \times 10^{-7}$, $1.8 \times 10^{-7}, 5.0 \times 10^{-6}, 4.5 \times 10^{-6}$, and $5.0 \times 10^{-5}$. In (b), from bottom to top, the values are $1-a / M=0,4.5 \times 10^{-8}, 1.25 \times 10^{-7}$, $1.8 \times 10^{-7}, 5.0 \times 10^{-6}, 4.5 \times 10^{-6}$, and $2.0 \times 10^{-1}$.

their extreme counterparts, but at late times approach those of nonextreme BHs (i.e., vanishing values). The closer the $\mathrm{BH}$ is to extremality, the longer it takes to lose its grown hair and achieve baldness. We examine the rate at which this behavior occurs below.
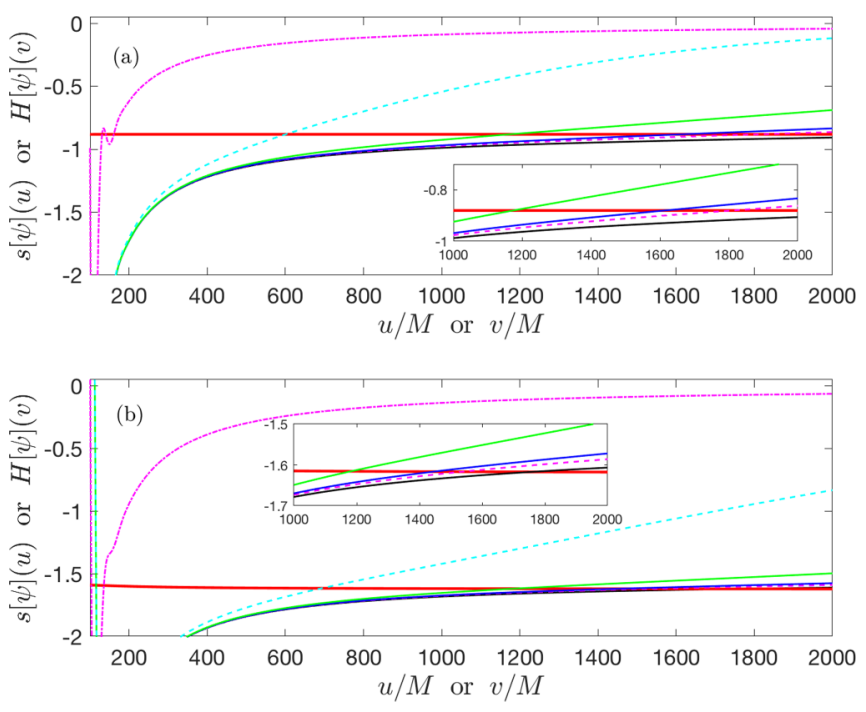

FIG. 4. The AAG hair $H[\psi](v)$ and its measurement at $\mathscr{I}^{+}$ $s[\psi](u)$ (in units of $M^{2}$ ) as functions of $v$ and $u$, respectively, for (a) ERN and (b) EK BHs, similarly to Figs. 1 and 2, correspondingly. Also shown is $s[\psi](u)$ for a number of values of $q / M$ and $a / M$, respectively. The insets magnify the late time period of near extremality. The values of $s[\psi](u)$ shown in either panel are the same as in Fig. 3. We show $H[\psi](v)$ by a nearly horizontal line (variability is unseen on the scale of the figure), at values of (a) $\sim 0.88$ and (b) $\sim-1.62$
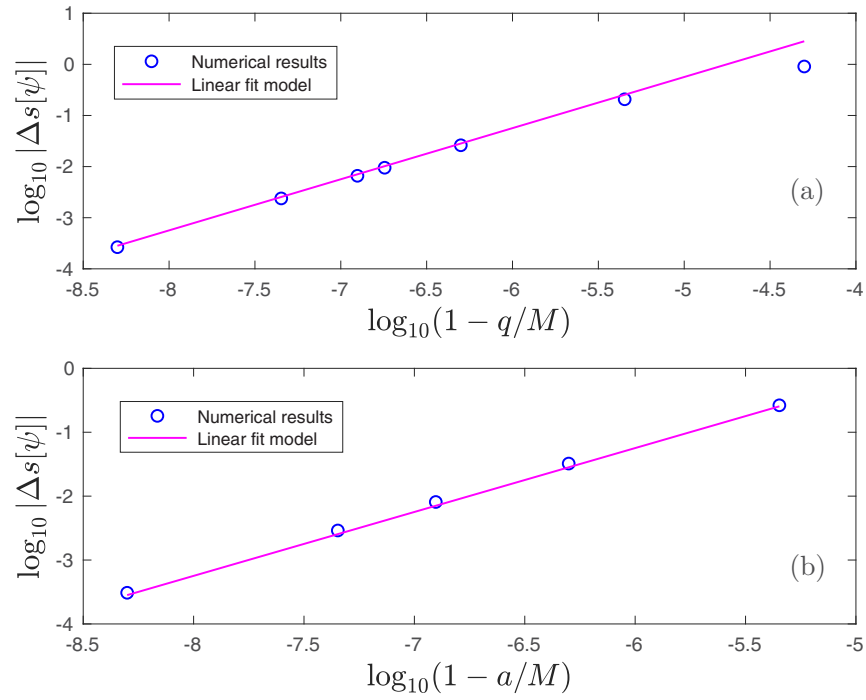

FIG. 5. Difference between the value of $s[\psi]$ for nearly extreme BHs and for an extreme BH, $\Delta s[\psi]$ (in units of $M^{2}$ ), as a function of (a) $1-q / M$ for NERN BHs at $u_{*}=1000 M$ and (b) $1-a / M$ for NEK BHs at $u_{*}=600 M$. The numerical data points are shown by circles, and the solid lines are linear best-fit lines with slopes of $0.997 \pm 0.010$ (NERN BHs) and $0.994 \pm 0.010$ (NEK BHs).

The behaviors shown above allow us to distinguish qualitatively between extreme, nonextreme, and nearly extreme BHs, where the third exhibits transient behaviors between the first and the second. We can obtain quantitative features of the transient nature of $s[\psi](u)$ for nearly extreme BHs by considering two complementary properties. First, consider a fixed value of retarded time $u=u_{*}$, and for a fixed value of $a / M$ or $q / M$ for Kerr or RN BHs, respectively, consider for NEK BHs $\Delta s[\psi](a / M):=\left.s[\psi]\right|_{u_{*}}(a / M)-\left.s[\psi]\right|_{u_{*}}(a / M=1)$ and an analogously defined function of $q / M$ for NERN BHs. In Fig. 5 we plot $\Delta s[\psi]$ as a function of $1-a / M$ for NEK BHs and as a function of $1-q / M$ for NERN BHs. For both cases we find that $\Delta s[\psi]$ is linear in the distance from extremality.

Second, we fix the value of $a / M$ or $q / M$. Define $\delta s[\psi](u ; a / M):=s[\psi](u ; a / M)-s[\psi](u ; a / M=1)$ for NEK BHs and an analogously defined function of $q / M$ for NERN BHs. In Figs. 6 and 7 we show $\delta s[\psi](u ; a / M)$ as functions of $u$ for NEK and NERN BHs, respectively. The difference between a nonextreme $\mathrm{BH}$ and its extreme counterpart is $O(1)$. For nearly extreme BHs the differences $\delta s[\psi](u ; a / M)$ or $\delta s[\psi](u ; q / M)$ are small at early times (dominated by quasinormal modes), but grow like $u^{2}$ at intermediate times. At sufficiently late retarded times, which increase with the greater closeness of the $\mathrm{BH}$ to extremality, the quadratic growth in retarded time slows down and $\delta s[\psi]$ approaches its nonextreme $\mathrm{BH}$ value asymptotically. For the computations we study in this work, the intermediate regime begins soon after the quasinormal ringing phase $(\sim 100 M)$ and then lasts for several hundred to thousands of $M$ depending on $a / M$.

We can now combine the previous results and suggest that for NEK BHs

$$
s[\psi]\left(u, \frac{a}{M}\right)=s[\psi]\left(u, \frac{a}{M}=1\right)+\mathscr{S}_{0}^{\mathrm{K}} u^{2}\left(1-\frac{a}{M}\right)
$$




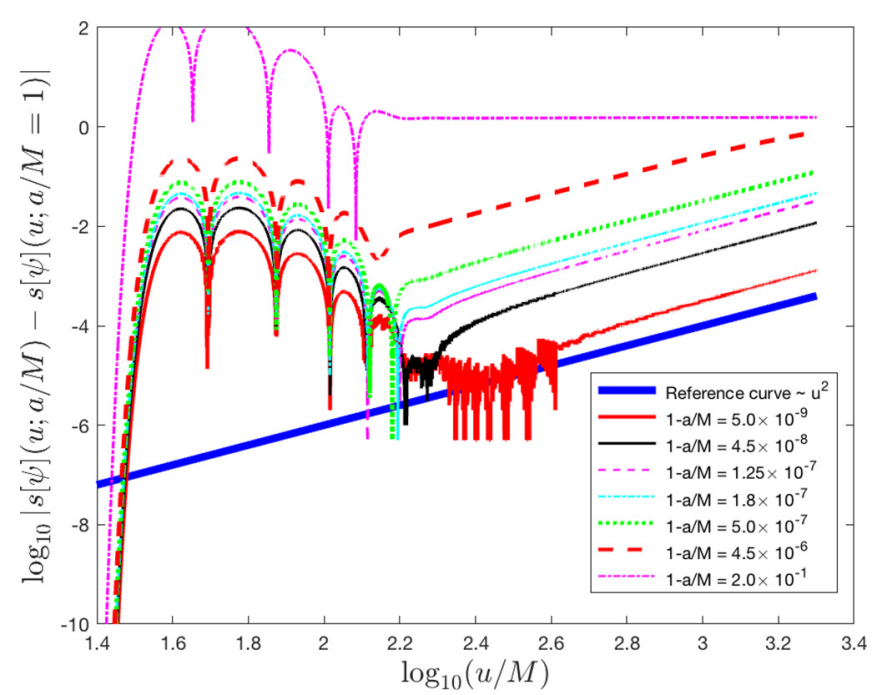

FIG. 6. Difference $\delta s[\psi]$ for different values of $a / M$ for Kerr BHs as functions of $u$. The reference line (thick solid curve) is $\sim u^{2}$.

and for NERN BHs

$$
s[\psi]\left(u, \frac{q}{M}\right)=s[\psi]\left(u, \frac{q}{M}=1\right)+\mathscr{S}_{0}^{\mathrm{RN}} u^{2}\left(1-\frac{q}{M}\right)
$$

at intermediate times. We find that the dimensionless coefficients $\mathscr{S}_{0}^{\mathrm{K}}=0.065 \pm 0.001$ and $\mathscr{S}_{0}^{\mathrm{RN}}=0.15 \pm 0.01$ for our choice of initial data.

\section{DISTINGUISHING EXTREME, NEAR-EXTREME, AND NONEXTREME RN OR KERR BLACK HOLES}

This deviation of nearly extreme BHs from their extremal counterparts allows for their observational identification by distant observers. Specifically, measurements at $\mathscr{I}^{+}$of a newly perturbed nearly extreme $\mathrm{BH}$ show initial growth of AAG hair. However, whereas for EK or ERN BHs where this hair is permanent, for nearly extreme BHs the length of the newly grown hair decreases initially quadratically in

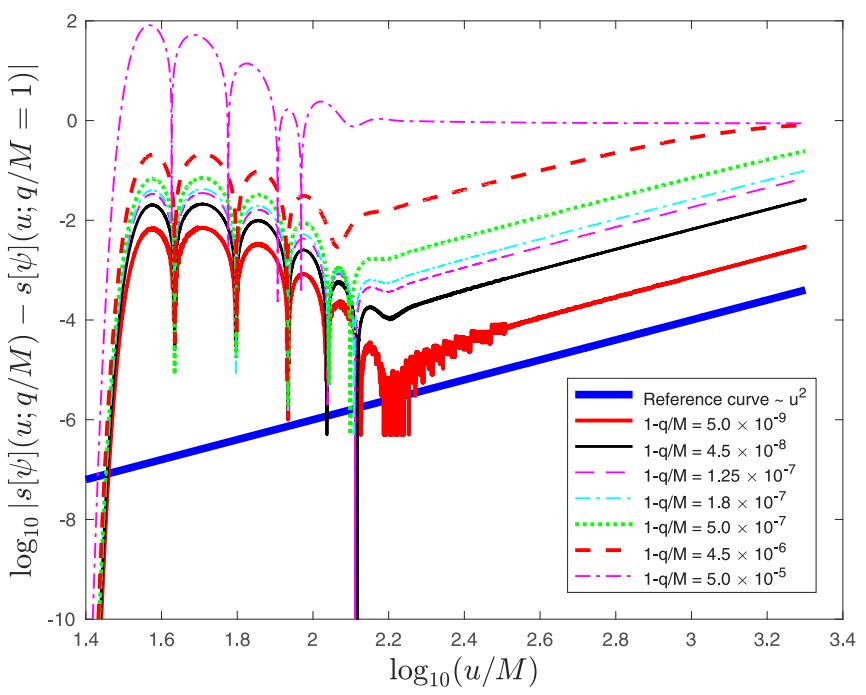

FIG. 7. Same as Fig. 6 for values of $q / M$ for RN BHs.
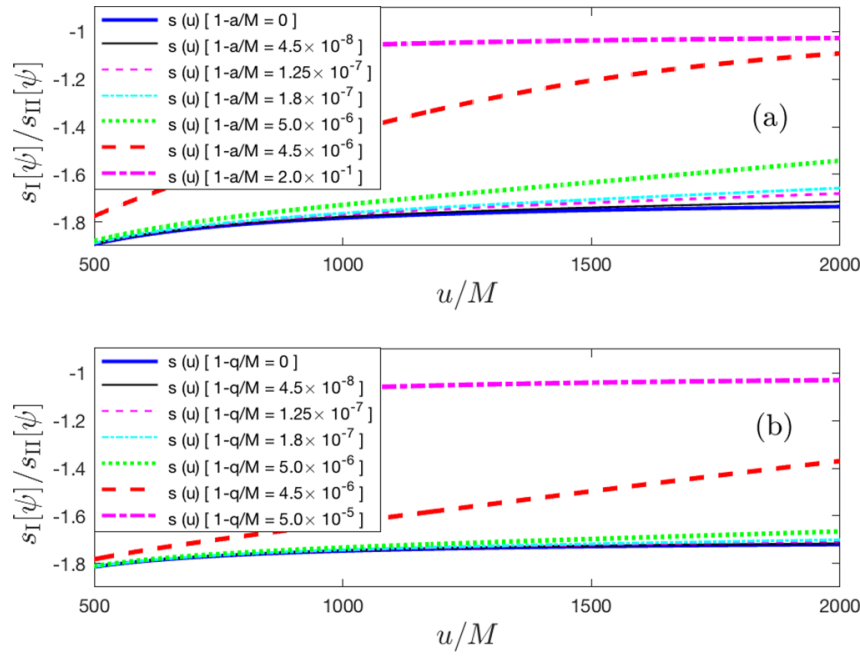

FIG. 8. Ratio $s_{\mathrm{I}}[\psi] / s_{\mathrm{II}}[\psi]$ as a function of $u$ for (a) Kerr BHs and (b) RN BHs.

time until its length becomes short and the rate at which the length shortens further slows down. Eventually, the nearly extreme $\mathrm{BH}$ becomes bald again like nonextreme BHs. The nearly extreme $\mathrm{BH}$ may repeat its hair regrowth attempts when it is perturbed again, but will never succeed for long: It is to eventually lose its regrown hair and become bald again.

We can gain additional insight into the transient behavior of NEK and NERN BHs by considering the relative contributions of the two terms in Eq. (1), $s_{\mathrm{I}}[\psi]$ and $s_{\mathrm{II}}[\psi]$. Figure 8 shows the ratio $s_{\mathrm{I}}[\psi] / s_{\mathrm{II}}[\psi]$ for EK and NEK BHs and for ERN and NERN BHs. For both EK and ERN BHs $s_{\mathrm{I}}[\psi] / s_{\mathrm{II}}[\psi] \rightarrow \sim-1.71$ as $u \rightarrow \infty$. For nonextreme BHs $s_{\mathrm{I}}[\psi] / s_{\mathrm{II}}[\psi] \rightarrow-1$ as $u \rightarrow \infty$. That is, each term in Eq. (1) approaches a nonzero constant for nonextreme BHs, yet their sum vanishes. For nearly extreme BHs Fig. 8 shows that at early times the ratio $s_{\mathrm{I}}[\psi] / s_{\mathrm{II}}[\psi]$ is close to its extreme $\mathrm{BH}$ counterpart, but at late times it approaches negative unity, as for nonextreme BHs. We again find that the closer the $\mathrm{BH}$ is to extremality, the longer it takes the ratio to get close to -1 .

Our analysis provides an answer to the question of when a BH is considered nearly extreme. As implied by Figs. 3, 4, 7, and 8 , when $1-q / M=5.0 \times 10^{-5}$ the transient scalar hair of the $\mathrm{BH}$ behaves as for nonextreme BHs. For $1-q / M=$ $4.5 \times 10^{-6}$ we already see typical transient behavior, the hallmark of nearly extreme BHs. This effect complements the signature that can be detected by the emission of gravitational waves from a plunge into a nearly extreme $\mathrm{BH}$ [15].

\section{ACKNOWLEDGMENTS}

The authors are indebted to Stefanos Aretakis for stimulating discussions. G.K. is grateful for support from National Science Foundation through Grant No. PHY-1701284 and Office of Naval Research, Defense University Research Instrumentation Program, Grant No. N00014181255. 
[1] C. A. R. Herdeiro and E. Radu, Int. J. Mod. Phys. D 24, 1542014 (2015).

[2] J. D. Bekenstein, Phys. Rev. Lett. 28, 452 (1972).

[3] J. D. Bekenstein, Phys. Rev. D 5, 1239 (1972).

[4] J. D. Bekenstein, Phys. Rev. D 5, 2403 (1972).

[5] C. Cao, Y.-X. Chen, and J.-L. Li, Commun. Theor. Phys. 53, 285 (2010).

[6] M. S. Volkov and D. V. Galtsov, Yad. Fiz. 51, 1171 (1990) [Sov. J. Nucl. Phys. 51, 747 (1990)]; P. Bizon, Phys. Rev. Lett. 64, 2844 (1990).

[7] L. Heisenberg, R. Kase, M. Minamitsuji, and S. Tsujikawa, Phys. Rev. D 96, 084049 (2017).

[8] S. Coleman, J. Preskill, and F. Wilczek, Nucl. Phys. B 378, 175 (1992).
[9] S. W. Hawking, M. J. Perry, and A. Strominger, Phys. Rev. Lett. 116, 231301 (2016).

[10] Y. Angelopoulos, S. Aretakis, and D. Gajic, Phys. Rev. Lett. 121, 131102 (2018).

[11] S. Aretakis, Commun. Math. Phys. 307, 17 (2011); Ann. Henri Poincare 12, 1491 (2011).

[12] A. Zenginoğlu, Classical Quantum Grav. 25, 145002 (2008).

[13] A. Zenginoğlu and G. Khanna, Phys. Rev. X 1, 021017 (2011).

[14] L. M. Burko, G. Khanna, and A. Zenginoğlu, Phys. Rev. D 93, 041501(R) (2016); 96, 129903(E) (2017).

[15] L. M. Burko and G. Khanna, Phys. Rev. D 94, 084049 (2016). 